\title{
Development of limb bone laminarity in the homing pigeon (Columba livia)
}

\author{
Rylee S McGuire ${ }^{1}$, Raffi M Ourfalian ${ }^{1,2}$, Kelly Ezell ${ }^{3}$, Andrew H Lee ${ }^{\text {Corresp. 1, 3, } 4}$ \\ 1 Arizona College of Osteopathic Medicine, Midwestern University, Glendale, Arizona, United States \\ 2 Kaiser Permanente Los Angeles Medical Center, Los Angeles, California, United States \\ 3 Department of Anatomy, College of Graduate Studies, Midwestern University, Glendale, Arizona, United States \\ 4 College of Veterinary Medicine, Midwestern University, Glendale, Arizona, United States \\ Corresponding Author: Andrew H Lee \\ Email address: alee712@gmail.com
}

Background. Birds show adaptations in limb bone shape that are associated with resisting locomotor loads. Whether comparable adaptations occur in the microstructure of avian cortical bone is less clear. One proposed microstructural adaptation is laminar bone in which the proportion of circumferentiallyoriented vascular canals (i.e., laminarity) is large. Previous work on adult birds shows elevated laminarity in specific limb elements of some taxa, presumably to resist torsion-induced shear strain during locomotion. However, more recent analyses using improved measurements in adult birds and bats reveal lower laminarity than expected in bones associated with torsional loading. Even so, there may still be support for the resistance hypothesis if laminarity increases with growth and locomotor maturation.

Methods. Here, we tested that hypothesis using a growth series of 17 homing pigeons (15-563 g). Torsional rigidity and laminarity of limb bones were measured from histological sections sampled from midshaft. Ontogenetic trends in laminarity were assessed using principal component analysis to reduce dimensionality followed by beta regression with a logit link function.

Results. We found that torsional rigidity of limb bones increases disproportionately with growth, consistent with rapid structural compensation associated with locomotor maturation. However, laminarity decreases with maturity, weakening the hypothesis that high laminarity is a flight adaptation at least in the pigeon. Instead, the histological results suggest that low laminarity, specifically the relative proportion of longitudinal canals aligned with peak principal strains, may better reflect the loading history of a bone. 


\section{Development of limb bone laminarity in the homing}

3 pigeon (Columba livia)

4

5

6 Rylee S. McGuire ${ }^{1}$, Raffi M. Ourfalian ${ }^{1,2}$, Kelly Ezell ${ }^{3}$, Andrew H. Lee 1,3,4,*

7

$8{ }^{1}$ Arizona College of Osteopathic Medicine, Midwestern University, Glendale, Arizona, United

9 States

$10 \quad{ }^{2}$ Kaiser Permanente Los Angeles Medical Center, Los Angeles, California, United States

$11{ }^{3}$ Department of Anatomy, College of Graduate Studies, Midwestern University, Glendale, 12 Arizona, United States

$13{ }^{4}$ College of Veterinary Medicine, Midwestern University, Glendale, Arizona, United States 14

* Corresponding Author: Andrew H. Lee

17

18

19 


\section{Abstract}

21 Background. Birds show adaptations in limb bone shape that are associated with resisting

22

23

24

locomotor loads. Whether comparable adaptations occur in the microstructure of avian cortical bone is less clear. One proposed microstructural adaptation is laminar bone in which the proportion of circumferentially-oriented vascular canals (i.e., laminarity) is large. Previous work on adult birds shows elevated laminarity in specific limb elements of some taxa, presumably to resist torsion-induced shear strain during locomotion. However, more recent analyses using improved measurements in adult birds and bats reveal lower laminarity than expected in bones associated with torsional loading. Even so, there may still be support for the resistance hypothesis if laminarity increases with growth and locomotor maturation.

Methods. Here, we tested that hypothesis using a growth series of 17 homing pigeons (15-563

g). Torsional rigidity and laminarity of limb bones were measured from histological sections sampled from midshaft. Ontogenetic trends in laminarity were assessed using principal component analysis to reduce dimensionality followed by beta regression with a logit link function.

Results. We found that torsional rigidity of limb bones increases disproportionately with growth, consistent with rapid structural compensation associated with locomotor maturation. However, laminarity decreases with maturity, weakening the hypothesis that high laminarity is a flight adaptation at least in the pigeon. Instead, the histological results suggest that low laminarity, specifically the relative proportion of longitudinal canals aligned with peak principal strains, may better reflect the loading history of a bone. 


\section{Introduction}

44 Laminar bone is a form of fibrolamellar bony tissue in which the primary vascular canal network

45 is organized into concentric interconnected layers (Francillon-Vieillot et al., 1990). It is

46 dominated by circumferential vascular canals (Currey, 1960; Francillon-Vieillot et al., 1990; de

47 Ricqlès et al., 1991; de Margerie, 2002; de Boef \& Larsson, 2007; Huttenlocker, Woodward \&

48 Hall, 2013), which have elongated profiles in transverse view that run approximately parallel to

49 the periosteal surface (Fig. 1). The proportion of laminar bone [laminarity (de Margerie, 2002)]

50 in many adult avian species appears elevated in specific limb bones such the humerus, ulna,

51 femur, and tibiotarsus (de Margerie et al., 2005). Theoretical modeling suggests that these limb

52 bones experience locomotor-induced torsion (i.e., by flapping in the humerus and ulna and by

53 walking in the femur and tibiotarsus) (Pennycuick, 1967). Indeed, in vivo bone strain

54 measurements confirm that torsional loading is substantial in the ulna of grounded but flapping

55 turkeys (Lanyon \& Rubin, 1984) and is dominant in the humerus of flying pigeons (Biewener \&

56 Dial, 1995). In addition, while walking, chickens and emus generate large torsional loads in the

57 femur and tibiotarsus (Biewener, Swartz \& Bertram, 1986; Carrano \& Biewener, 1999; Main \&

58 Biewener, 2007). If these loading patterns are similar across birds, then the elevated laminarity

59 observed in humeri, ulnae, femora, and tibiotarsi of many avian species may be a general feature

60 of limb bones loaded habitually in torsion (de Margerie et al., 2005).

61 A purely biomechanical explanation for laminarity, however, remains problematic.

62 Contrary to the laminarity hypothesis, the only two species with detailed measurements of flight-

63 induced torsion [Columba livia (Biewener \& Dial, 1995) and Pteropus poliocephalus (Swartz,

64 Bennett \& Carrier, 1992)] actually have negligible to low laminarity in the adult humerus

65 (Bennett \& Forwood, 2010; Lee \& Simons, 2015; Pratt et al., 2018). Furthermore, any form of

66 vascularization is substantially reduced in the superficial cortex, where maximum flight-induced 
67 torsional and bending loads are predicted (Pennycuick, 1967; Carter \& Spengler, 1978; Craig,

68 2000). The relatively avascular parallel-fibered (or lamellar) bone of the superficial cortex

69 reflects growth attenuation that occurs in birds and mammals as they approach adult-size (e.g.,

70 de Margerie, Cubo \& Castanet, 2002; Castanet et al., 2004; Ponton et al., 2004; Main, 2007;

71 Kuehn et al., 2019). Once adult size is reached, normal locomotor-induced loads are not able to

72 stimulate deposition of new bone along the superficial cortex, laminar or otherwise (e.g., Bennett

73 \& Forwood, 2010). These observations suggest limits to where and when laminar bone can form.

74 Therefore, further sampling across the development of the pigeon is needed to clarify the extent

75 of laminar bone in juveniles.

76 Postnatal development in the pigeon is altricial (Starck \& Ricklefs, 1998). Juveniles are

77 flightless and nest-bound for most of the postnatal growth period (Vriends \& Erskine, 2005;

78 Coles, 2007; Liang et al., 2018). Only when nearly full-grown do they become powerful fliers

79 (Tobalske \& Dial, 1996). Thus, the pigeon is ideal to examine rapid structural compensation in

80 the limb skeleton during locomotor transition. Because second moment of area at midshaft,

81 which indicates bone wall thickness, increases disproportionately with body mass in the altricial-

82 developing wings of the California gull (Carrier \& Leon, 1990) and mallard (Dial \& Carrier,

83 2012), so we expect a similar increase in the altricial-developing forelimb and hindlimb bones of

84 the pigeon. Specifically, polar section modulus, which is related to second moment of area and

85 proportional to torsional strength and rigidity at midshaft (Ruff, 2002; Young, Fernández \&

86 Fleagle, 2010; Ruff et al., 2013), should scale with positive ontogenetic allometry. Furthermore,

87 if laminarity is a reflection of locomotor-induced torsion (de Margerie et al., 2005), then it

88 should increase dramatically with skeletal (and locomotor) maturity.

89

90 Materials \& Methods 


\section{Sampling and histology}

92 We acquired salvaged carcasses of 17 homing pigeons (Stromberg's Chicks \& Game Birds

93 Unlimited; Pine River, Minnesota, USA). The sample comprises a postnatal growth series of 94 known mass $(15-563 \mathrm{~g})$ (Table S1). Although the age range of the sample is $0-9$ weeks, the

95 precise age of death for most individuals was not recorded. The right fore- and hindlimb of each

96 individual was dissected to reveal the humerus, radius, ulna, femur, and tibiotarsus (Fig. 2A).

97 The length of each element was measured (Tables S2-S6), and a 1-cm mid-diaphyseal block

98 from each bone was excised using a rotary tool (Dremel 4000; Dremel, Mt. Prospect, Illinois,

99 USA). We followed an established protocol for preparing plastic-embedded undecalcified bone

100 (Lee \& Simons, 2015). Specifically, two transverse 700- $\mu \mathrm{m}$ wafers were cut from each specimen

101 at mid-diaphysis using a precision saw (Isomet 1000; Buehler, Lake Bluff, Illinois, USA).

102 Wafers were mounted (Gorilla Epoxy; Gorilla Glue Inc., Cincinnati, Ohio, USA) to glass slides

103 and thinned to $100 \pm 10 \mu \mathrm{m}$ using a grinder/polisher (Metaserv 250; Buehler, Lake Bluff,

104 Illinois, USA).

105

106 Imaging

107 Sections were acid-etched and stained with toluidine blue (Eurell \& Sterchi, 1994) to improve

108 contrast of in-plane primary vascular canals (Fig. 2B). The stain also highlights secondary

109 osteons (specifically cement lines) and their (Haversian) canals, which are traditionally excluded

110 from measurements of laminarity (de Margerie, 2002). Whole section images pre- and post-

111 staining were captured with a motorized microscope (Ni-U; Nikon, Tokyo, Japan) as previously

112 described by Lee and Simons (2015). Once imaging was completed, each section was mounted

113 (Permount; Fisher Scientific, Hampton, New Hampshire, USA) with a glass coverslip (\#1; Fisher 
114 Scientific, Hampton, New Hampshire, USA) for preservation (Lee \& Simons, 2015). Sections

115 are housed in the Arizona Research Collection for Integrative Vertebrate Education and Study 116 (ARCIVES) at Midwestern University.

117

\section{Bone profiles}

119 A bone profile was prepared from each montage following procedures described by Lee and 120 Simons (2015). Specifically, montages were sharpened in Photoshop (CS5; Adobe, San Jose, 121 California, USA) with the "Unsharp Mask" filter (5 px). The area bounded between the 122 periosteal and endosteal surfaces was filled with white to represent bone. The surrounding non123 bone area as well as in-plane vascular canals and resorption spaces were filled with black. Bone 124 profiles were exported to ImageJ (1.51d; National Institutes of Health, Bethesda, Maryland, 125 USA) for further analysis. We measured the periosteal circumference and vascular porosity (ratio 126 of in-plane primary and secondary vascular area to total cortical area) of each bone profile 127 (Tables S2-S6). Montages and bone profiles can be previewed at the Paleohistology Repository 128 (Lee \& O'Connor, 2013) and downloaded at Dryad.

129

130

131 132 134 135 136

\section{Ontogenetic scaling of polar section modulus}

Bone profiles were imported into ImageJ, and the BoneJ plugin v1.4.1 (Doube et al., 2010) was used to calculate geometric properties such as second moment of area $(I)$, polar moment of area $(J)$, and polar section modulus $\left(Z_{p}\right)$. These properties describe the distribution of material around the centroid of a given bone section and are inversely proportional to bending stress, overall (bending and torsional) stress, and maximum overall stress, respectively (e.g., Schoenau et al., 2001; Ruff, 2002; Habib \& Ruff, 2008; Young, Fernández \& Fleagle, 2010; Hedrick et al., 
137 2020). In other words, a bone section with large $I, J$, and $Z_{p}$ experience less stress for a given

138 load, giving it greater strength and rigidity to withstand larger loads before failure. Because those

139 properties are closely related, we only present the $Z_{p}$ data (Tables S2-S6), which are the most

140 relevant to test the torsional resistance hypothesis.

$141 \quad Z_{p}$ is proportional to the maximum torsional stress that occurs at the outermost surface of

142 a bone loaded in pure torsion (Craig, 2000). It is calculated by taking the ratio of $J$ and the

143 maximum distance between the centroid and the periosteal surface $\left(r_{\max }\right)$, where failure is most

144 likely to occur. BoneJ calculates $Z_{p}$ without assuming circular or elliptical geometry by applying

145 the following general relationship (Eq. 1) to pixel data in the actual bone profiles:

$$
Z_{p}=\frac{J}{r_{\max }}=\frac{\int r^{2} d A}{r_{\max }},
$$

147 where $J$ is the integral sum of the area of each pixel $d A\left(2.36 \mathrm{e}-7 \mathrm{~mm}^{2}\right)$ representing bone that is a 148 distance $r$ from the centroid, and $r_{\max }$ is the maximum distance between the centroid and the 149 periosteal surface (Doube et al., 2010). $Z_{p}$ is an appropriate proxy for torsional strength and 150 rigidity when the cross section of a long bone is nearly circular (Craig, 2000). To test the 151 suitability of this proxy to each cross section, we used BoneJ to calculate the aspect ratio $152\left(I_{\max } / I_{\min }\right)$, which equals 1 in a circular cross section. Values for the bone sections range from 1531.03 to 1.80 (Tables S2-S6). When compared to a reference figure (Daegling, 2002), the values 154 of $I_{\max } / I_{\min }$ in our sample suggest errors in torsional rigidity less than $6.7 \%$. Therefore, we find no 155 major problem in using this proxy.

156 Using R (R Core Team, 2019), $Z_{p}$ and body mass were $\log _{10}$-transformed to linearize 157 their relationship before performing separate Type I linear regression for each element. The 158 scaling coefficients $(b)$ and exponents $(a)$ of the regression analysis are presented in Table 1.

159 Because $Z_{p}$ and body mass $(M)$ are each proportional to length ${ }^{3}$, allometric scaling of the log-log 
160 model, $\log _{10}\left(Z_{p}\right)=\log _{10}(b)+a \log _{10}(M)$, was inferred if the $95 \%$ confidence interval of the

161 scaling exponent (a) excluded the value of 1 (isometry). Note that we chose to use Type I instead

162 of Type II (RMA) regression for two reasons. First, $Z_{p}$ and body mass were measured precisely

163 with low error. Second and more importantly, there is natural individual variation in $Z_{p}$ for a

164 given body mass. Such variation, whether influenced by intrinsic or extrinsic factors, is known to

165 weaken Type II regression, which has a tendency to detect a steeper relationship than actually

166 exists (Kilmer \& Rodríguez, 2017).

167

168 Laminarity index. We classified each vascular canal into one of four discrete categories of 169 orientation (longitudinal, radial, oblique, and circumferential). Although, the laminarity index 170 (LI) was originally defined by de Margerie (2002) as the proportionate area of circumferential 171 canals relative to the total area of vascular canals, we used a subsequent re-definition in which LI 172 is the number of circumferential canals divided by the total number of canals (Simons \& 173 O’Connor, 2012; Legendre et al., 2014; Lee \& Simons, 2015). As such LI is a proportion and 174 ranges from 0 (absence of circumferential canals) to 1 (ubiquity of circumferential canals). 175 We adopted a recently published method to quantify LI (Lee \& Simons, 2015).

176 Specifically, instead of counting every canal in an image of a bone section, we sampled LI from 177 four anatomical octants and calculated mean LI representing approximately $50 \%$ of the total 178 canals in a given section. The image of each section was divided into octants using Photoshop 179 (Fig. 2B), and the four octants representing cardinal anatomical positions (i.e., cranial, caudal, 180 dorsal, and ventral for the wing elements; cranial, caudal, medial, and lateral for the hindlimb 181 elements) were extracted for analysis. 

surface, however, is curved in most bone cortices (Fig. 2C). Consequently, the local tangent varies across a curved cortex and requires repeated referencing to measure canal orientation. We

185 186

used the method originally described by Lee and Simons (2015) to increase throughput and minimize error by straightening the curvature of each octant (Fig. 2D) using the "Straighten" function in ImageJ. Minimal distortion was verified by comparing octants overlaid with test angles before and after straightening.

We adopted the method by de Boef and Larsson (2007) to approximate the sectional profile of each primary vascular canal with a best-fitting ellipse using ImageJ. Aspect ratio and angle between the periosteal surface and major axis of ellipses were measured. To relate these measurements to canal orientation, we followed criteria originally proposed by de Margerie (2002): (1) longitudinal canals have a roughly circular profile with an aspect ratio of less than 3 ; (2) circumferential canals have a major axis that is roughly parallel to the periosteal surface $\left(0^{\circ} \pm\right.$ $22.5^{\circ}$ ); (3) radial canals have a major axis that is roughly perpendicular to the periosteal surface $\left(90^{\circ} \pm 22.5^{\circ}\right)$; and (4) oblique canals have an aspect ratio greater than 3 with a major axis oriented between $22.5^{\circ}$ and $67.5^{\circ}$ to the periosteal surface (Fig. 2E). Any canal that branches was divided at the node, and the orientation of each subdivided canal was estimated using the methodology as described above.

The ellipse-fitting method is appropriate as long as canals are generally cylindrical. They tend to be in cortical bone (Cooper et al., 2003, 2011; Pratt \& Cooper, 2017), which ranges in vascular porosity from 0 - 30\% (Carter \& Spengler, 1978; Zioupos, Cook \& Hutchinson, 2008). MicroCT inspection suggests this assumption is reasonable for avian cortical bone (Fig. 1). However, in cancellous bone (Carter \& Spengler, 1978; Zioupos, Cook \& Hutchinson, 2008) 
205 with vascular porosity greater than $30 \%$, canals are too irregular to approximate with the ellipse-

206 fitting method. Consequently, we measured canal orientation only in bone sections with porosity

207 less than or equal to 30\% (Tables S2-S6) and excluded the youngest specimens (MWU263,

208 MWU 261, MWU 260, and MWU 267) from further analyses of laminarity.

209

210 Robust principal component analysis and beta regression

211 In this study, we had to address the issue of multicollinearity among our explanatory variables:

212 mass, bone length, and $Z p$. Principal component analysis (PCA) enables the formation of new,

213 uncorrelated predictors (principal components) through linear combinations of the original

214 variables. As such, we were able to resolve the issue of multicollinearity while still being able to

215 assess the effect of each variable (Fekedulegn et al., 2002). PCA, however, is known to be highly

216 sensitive to non-normal data. Therefore, we used robust PCA, which is appropriate for skewed

217 data (Hubert, Rousseeuw \& Verdonck, 2009), as implemented by the R package "rospca"

218 (Reynkens, 2018). We standardized mass, bone length, and $Z p$ by median and median absolute

219 deviation with the function "RobScale" (Signorell, 2019) in R. Data were grouped by

220 homologous element, and a separate robust PCA was performed for each element (Table 2).

221

We used regression analysis to relate the minimum number of principal components

222 (PCs) that account for at least 95\% of the variation in the original variables with laminarity.

223 However, laminarity index (LI) values do not satisfy assumptions required of traditional linear

224 regression because they are not normally distributed and are bounded between 0 and 1. To

225 overcome these problems, we used the following beta regression model with a logit link function

226 (Eq. 2) to connect mean LI to the linear predictor:

227

$$
\operatorname{logit}(\mathrm{LI})=\ln \left(\frac{\mathrm{LI}}{1-\mathrm{LI}}\right)=\beta_{0}+\beta_{\mathrm{i}} P C_{\mathrm{i}}+\ldots+\beta_{\mathrm{k}} P C_{\mathrm{k}}, \mathrm{i}=1, \ldots, \mathrm{k}
$$


228 where logit(LI) is the logit link function for the mean of LI and the linear predictor is defined by

$229 P C_{\mathrm{i}}, \ldots, P C_{\mathrm{k}}$ as the scores of each principal component, $\beta_{0}$ as the intercept, $\beta_{\mathrm{i}}, \ldots, \beta_{\mathrm{k}}$ as

230 coefficients corresponding to each principal component, and $\mathrm{k}$ as the number of principal

231 components (Ferrari \& Cribari-Neto, 2004). No 0- or 1-values were observed in laminarity, so

232 we did not need to apply either a transformation to slightly shift boundary values or sophisticated

233 zero-one inflated beta regression (Smithson \& Verkuilen, 2006; Douma \& Weedon, 2019).

234 Instead, traditional beta regression was performed with the R package "gamlss" (Rigby \&

235 Stasinopoulos, 2005). To ease interpretation of fitted models on the scale of observed laminarity

$236(0,1)$, the linear predictor was transformed using the inverse logit function. Thus, the resulting

237 expression (Eq. 3) becomes a relationship between absolute changes in PC scores and mean LI:

$$
\mathrm{LI}=\frac{e^{\eta}}{1+e^{\eta}}
$$

where the linear predictor $\eta$ is $\beta_{0}+\beta_{\mathrm{i}} P C_{\mathrm{i}}+\ldots+\beta_{\mathrm{k}} P C_{\mathrm{k}}$ (Douma \& Weedon, 2019).

240 Standardized coefficients of the original predictors (mass, bone length, and $Z_{p}$ ) were calculated

241 by multiplying the vector of $\beta$-coefficients by the matrix of eigenvectors from the PCA

242 (Fekedulegn et al., 2002: Eq. 23).. 


\section{Results}

\section{Histological description}

246 At mid-diaphysis, the cortical bone in the limbs of the sampled pigeons become increasingly

247 compact with growth (Tables S2-S6). In very young individuals ranging from 0-2 weeks (0-

$24840 \%$ of adult mass), bone walls are relatively thick and largely cancellous (porosity $>30 \%$ ) with

249 irregular vascular spaces. That cancellous structure is consistent with rapidly-growing juvenile

250 bone as seen in other avian species (de Margerie et al., 2004). Older individuals show compact

251 bone with vascular canals. For each bone, peak laminarity (i.e., proportion of circumferentially

252 oriented canals) occurs in pre-fledge juveniles (Figs. 2 and 3) that range approximately from 2-4

253 weeks of age and $40-70 \%$ of adult mass. These juveniles also show large disparity in laminarity

254 with much greater values occurring in the humerus, ulna, and femur than in the radius and

255 tibiotarsus. In post-fledge individuals (4-9 weeks of age and $70-100 \%$ of adult mass), the outer

256 bone cortex is poorly vascularized with a subperiosteal region of avascular parallel-fibered bone

257 in the oldest individuals of the sample (Fig. 3A-J). The remaining deep cortex is highly

258 vascularized, but canals are predominantly longitudinal with only slight differences in laminarity

259 among elements (Fig. 3A-J). Secondary osteons are generally uncommon in the sample. The

260 notable exception is the tibiotarsus in which secondary osteons are abundant in the deep cortex

261 towards the end of the sampled growth period.

262

\section{$263 Z p$ scaling analysis}

264 The polar section modulus $\left(Z_{p}\right)$ of the humerus, radius, ulna, femur, and tibiotarsus increases

265 with growth (Tables S2-S6). When scaled to $\log _{10}$-transformed body mass, $\log _{10}$-transformed $Z p$

266 shows significant positive allometry for all five sampled elements (Fig. 4) with 95\% confidence

267 intervals of the allometric exponents (a) each exceeding and excluding the isometric value of 1 
268 (Table 1). The allometric exponent of the tibiotarsus is slightly (but not significantly) shallower 269 than those of the other elements, in part reflecting the relatively large size of the tibiotarsus at 270 hatch (Tables 1, S2-6).

271

\section{Robust principal component analysis}

273 Robust principal component analysis is generally consistent across the five limb elements (Table 274 2). PC1 captures at least $95 \%$ of the variance in the original predictors: $98 \%$ for the humerus, $27595 \%$ for the radius, $97 \%$ for the ulna, $98 \%$ for the femur, and $96 \%$ for the tibiotarsus. We ignored 276 the residual variance (approximately $2-5 \%$ ) that is absorbed by PC2 and PC3, thereby reducing 277 data dimensionality from three components to one. Mass, $Z_{p}$ (torsional rigidity), and bone length 278 each have positive loadings on PC1. $Z_{p}$ and bone length have strong effects on PC1, but 279 dominance varies depending on the element. In the humerus and femur, $Z_{p}$ is dominant or 280 codominant with bone length, respectively, whereas in the remaining elements, length is 281 dominant (Table 2). Taken together, the loadings are consistent with PC1 representing an 282 ontogenetic axis. Small PC1 scores are associated with juvenile features (small mass with short 283 bones that are relatively compliant to torsion), whereas large PC1 scores are associated with 284 adult features (large mass with long bones that are relatively rigid to torsion).

\section{Beta regression}

287 The fitted beta regression models with a logit link have significant $\beta$-coefficients (Table 3). They 288 predict an absolute one-unit increase in PC1 leads to a relative change by a factor of $\exp \left(\beta_{1}\right)$ in 289 the ratio of laminarity (LI) to non-laminarity (1-LI). Thus, there is a relative decrease in the ratio 290 of laminarity to non-laminarity by: $22 \%$ in the humerus $[\exp (-0.249)-1] ; 22 \%$ in the ulna [exp(- 
$2910.245)-1] ; 18 \%$ in the radius $[\exp (-0.197)-1] ; 22 \%$ in the femur $[\exp (-0.244)-1] ;$ and $13 \%$ in

292 the tibiotarsus $[\exp (-0.137)-1]($ Table 3$)$. To better interpret these results as absolute changes in

293 laminarity, the fitted beta regression model for each element was back-transformed with the

294 inverse logit function and plotted. Each element shows a significant negative non-linear

295 relationship between LI and PC1 (Fig. 5). Two groups are apparent. The first group, consisting

296 of humerus, ulna, and femur, is characterized by relatively strong goodness-of-fit (pseudo- $\mathrm{R}^{2}$

297 exceeds 0.70), relatively positive intercept (i.e., larger mean LI across ontogeny as calculated by

298 Eq. 3), and steep negative slope. In contrast, the second group, consisting of radius and

299 tibiotarsus, is characterized by relatively weak goodness-of-fit (pseudo- $\mathrm{R}^{2}<0.55$ ), relatively

300 negative intercept (i.e., smaller mean LI across ontogeny), and shallow negative slope. Although

301 laminarity generally decreases with ontogeny in the homing pigeon, laminarity in the radius and

302 tibiotarsus may be influenced by additional unknown factors.

303 To express the PC1 coefficient in terms of the original predictors (mass, length, and $Z_{p}$ ),

304 the eigenvectors and coefficient of PC1 were multiplied by each other (Fekedulegn et al., 2002:

305 Eq. 23). The result is a set of principal component estimators of the standardized effects of the

306 original predictor on laminarity (Table 3). In all but two elements, laminarity is most influenced

307 by the effect of bone length, which is at least 1.5 times greater than the effects of either $Z_{p}$ or

308 mass. Different patterns occur in the humerus and femur. In the former, the strongest effect is $Z_{p}$,

309 and in the latter, the effects of bone length and $Z_{p}$ are similar in strength. Contrary to

310 expectations, none of the standardized coefficients are positive indicating that, at least in the

311 homing pigeon, growth in mass, bone length, and torsional rigidity have inverse effects on

312 laminarity. 


\section{Discussion}

\section{Positive allometric growth of torsional rigidity at midshaft}

316 This study demonstrates that some structural changes in midshaft cortical bone are predictable

317 responses to delayed locomotor development. Juvenile pigeons receive extended parental care 318 within protected nests and have limited mobility for over half of their postnatal growth period 319 (Levi, 1962; Janiga \& Kocian, 1985; Johnston \& Janiga, 1995; Vriends \& Erskine, 2005; Liang 320 et al., 2018). Under those conditions, selection for relatively robust juvenile limbs is likely 321 relaxed and reflected in "underbuilt" midshaft cortices. As juvenile pigeons approach adult size 322 and become fully mobile, torsional rigidity increases rapidly in forelimb and hindlimb bones as 323 indicated by significant positive ontogenetic allometry of midshaft $Z_{p}$ (Fig. 4). forelimb bones. Like pigeons, larids and mallards hatch with altricial wings and are unable to fly until juveniles are nearly adult size. Late development of wings involves accelerated growth in pectoral muscle mass, wing surface area, and breaking strength of bones. The latter is attributed in part to disproportionately large increases to forelimb bone midshaft width and second moment of area (Carrier \& Leon, 1990; Bennett, 2008; Dial \& Carrier, 2012). Late investment in wings may allow resource allocation to develop other metabolically expensive organ systems first (e.g., digestive, nervous, cardiovascular, and integumentary) (Carrier \& Leon, 1990; Johnston \& Janiga, 1995; Dial \& Carrier, 2012; Altimiras et al., 2017). A tentative conclusion from these results is that altriciality and positive allometric growth are coupled at least in avian wing development. However, we are aware that semi-intensively farmed Japanese quail from China show positive allometric growth of midshaft width in forelimb bones (Ren, Wang \& Zhang, 2016), which is odd given the early locomotor capabilities of the wings. Positive allometry of 
337 midshaft width was also found in the femur and tibiotarsus, the latter being inconsistent with

338 results from the closely-related chicken (Biewener \& Bertram, 1994). Because the analysis on

339 those Japanese quail used Type II regression, which has tendency to upward bias allometric

340 slopes (Kilmer \& Rodríguez, 2017), we suspect that negative allometry or isometry was

341 mistaken for positive allometry. In any event, additional sampling is needed to test whether avian

342 taxa with precocial wings capable of flight shortly after hatching [e.g., the Megapodidae (Dial \&

343 Jackson, 2011)] show negative allometric or isometric growth of forelimb bones, presumably to

344 keep breaking loads relatively constant as they function in flight while still growing.

345 Unlike the pigeon, the California gull and mallard have hindlimbs that are functional for

346 walking or swimming shortly after hatching. Their juveniles have relatively robust hindlimb

347 bones and generally experience negative allometric or isometric growth in midshaft width and

348 second moment of area, presumably to maintain locomotor performance and bone strength

349 comparable to those of adults ( Carrier \& Leon, 1990; Dial \& Carrier, 2012). The glaucous-

350 winged gull also has functional hindlimbs at hatching. However, results from an ontogenetic

351 study are not presented in terms of allometric scaling to body mass (Hayward et al., 2009).

352 Instead, they show that sigmoidal growth in midshaft width in hindlimb bones peaks relatively

353 early and is completed before forelimb bones. That pattern is consistent with negative allometry

354 and supports the hypothesis that growth in limb bone geometry reflects locomotor needs during 355 development.

356 Although distinct allometric growth trajectories of hindlimb bones may, to an extent,

357 indicate locomotor ability in juveniles, there are at least two examples that caution against

358 oversimplification. Like previously discussed larids (Carrier \& Leon, 1990; Hayward et al.,

359 2009), the Black noddy shows negative allometric growth in second moment of area of the 
360 tibiotarsus (Bennett, 2008). However, the Black noddy is tree- or cliff-nesting, which is a

361 behavior that provides refuge to juveniles but limits their locomotion during the growth period.

362 Nevertheless, juveniles still grow tibiotarsii with relatively thick cortices that compensate for low

363 mineralization to maintain bending strength and stiffness comparable to adults (Bennett, 2008).

364 Allocation of resources to develop relatively robust locomotor-capable hindlimb bones in nest-

365 bound juveniles is counterintuitive, and negative allometric growth in hindlimb bones may

366 simply be a relic of ancestral selection for locomotor ability in juveniles shared among larids

367 (Bennett, 2008).

368 Emus are capable of walking within two days after hatching (Sales, 2007). Yet femora

369 and tibiotarsii of chicks and early juveniles tend to be "underbuilt" relative to adults as indicated

370 by positive allometry in polar moment of area (Main \& Biewener, 2007). This ontogenetic

371 scaling relationship differs from the negative allometric growth reported in some precocial

372 mammals (e.g., jackrabbit and goat), which increases bone safety factors in juveniles when

373 performing locomotion similar to adults (e.g., Carrier, 1983; Main \& Biewener, 2004, 2007). The

374 reason for the difference in hindlimb bone growth allometry among precocial species is not clear,

375 but captivity is not likely a strong factor. Although emus and goats were studied under similar

376 captive conditions with free access to exercise, they show divergent allometric patterns in

377 hindlimb bone growth of polar moment of area (Main \& Biewener, 2004, 2007). Differences in

378 behavioral ecology was noted as a potential explanation; when compared to young goats,

379 juvenile emu spend more time foraging at slower speeds instead of evading threats at faster

380 speeds. Therefore, the "underbuilt" cortices of hindlimb bones in juvenile emu may reflect

381 relaxed selection for high-performance locomotion (Main \& Biewener, 2006). In a similar way, 
382 juvenile pigeons may experience relaxed selection for torsionally rigid limb bones given that

383 they delay development of aerial and terrestrial locomotion until nearly full-grown.

384

385 Limb bone laminarity decreases with maturity

386 Contrary to previous work on domestic turkey (Skedros \& Hunt, 2004) and emu (Kuehn et al.,

387 2019), our results in the homing pigeon do not support the hypothesis that laminarity increases as

388 a developmental response to locomotor-induced torsion. Unlike midshaft cortical geometry,

389 which appears to respond to locomotor maturation by growing progressively rigid to torsion,

390 laminarity decreases such that locomotor-capable adults have low values $(2.4-15.8 \%$; Figs. 3

391 and 5). Adults have low laminarity bone in part because the growth of the medullary cavity

392 resorbs all record of earlier juvenile bone with higher laminarity. The remaining cortex with

393 fewer circumferential canals (i.e., lower laminarity) reflects gradual reduction in periosteal

394 deposition with skeletal maturation (Supplemental Videos S1-5). According to the laminarity

395 hypothesis, low laminarity is a feature of limb bones adapted to locomotion involving reduced

396 torsional loading. For example, low laminarity in the forelimb bones of birds with long and

397 narrow wings may reflect habitual loading in bending rather than torsion (de Margerie et al.,

398 2005; Simons \& O'Connor, 2012). The juvenile and young adult homing pigeons in our sample

399 were raised in enclosed lofts with minimal crowding and free access to exercise, but without in

400 vivo bone strain data, we recognize that biomechanical inferences based on our sample of

401 homing pigeons remain speculation. Nevertheless, there is consilience between cross-sectional

402 geometry from the current study and in vivo off-axis principal strains from other studies of adult

403 birds, including feral pigeons (Lanyon \& Rubin, 1984; Biewener, Swartz \& Bertram, 1986;

404 Biewener \& Dial, 1995; Carrano \& Biewener, 1999; Main \& Biewener, 2007), that suggests 
405 substantial locomotor-induced torsion at least in the humerus, ulna, femur, and tibiotarsus. A 406 circumspect conclusion drawn from limited data is that bones adapted to resist torsional loading 407 do not necessarily increase laminarity during development.

408 Recent work on adult feral pigeons reveals "high" laminarity at least in the humerus 409 (Skedros \& Doutré, 2019). The contrasting results suggest that laminarity in homing pigeons 410 may not be as representative of the wild-type as assumed by the present study. However, the 411 assessment of "high" laminarity in feral pigeons is based on casual inspection rather than a 412 canal-by-canal count. Therefore, we cannot exclude the possibility that it is an overestimate. 413 Indeed, when canal-by-canal counts are performed in other avian taxa either with histological 414 (Lee \& Simons, 2015) or computed tomographic methods (Pratt \& Cooper, 2017), laminarity 415 that is previously reported to be "high" changes to "low" (Pratt et al., 2018). Similarly, we expect 416 a reassessment to find low laminarity in adult feral pigeons.

417 The focus on circumferential canals in the laminarity hypothesis needs better

418 biomechanical justification. If vascular canals align circumferentially to reflect torsional loading 419 on a given bone, then the canals should be roughly concentric with an approximate angle of $45^{\circ}$ 420 from the longitudinal axis. This angle corresponds to the orientation of peak principal strain 421 during pure torsional loading (Craig, 2000). Empirical data from in vivo bone strain experiments 422 on birds during locomotion demonstrate that the orientation of peak principal strain varies with 423 limb bone and species but is still reasonably close to $45^{\circ}$ (Biewener, Swartz \& Bertram, 1986;

424 Biewener \& Dial, 1995; Carrano \& Biewener, 1999; Main \& Biewener, 2007). In contrast, 425 circumferential canals are defined as "in-plane” features (de Margerie, 2002) and are aligned 426 closer to the transverse plane of section. Because vascular canals are approximately cylindrical 427 (Fig. 1), orientation can be estimated from a histological section by applying the in-plane aspect 
428 ratio of the canal (i.e., greater than 3: de Margerie, 2002) to a trigonometric equation (de Boef \&

429 Larsson, 2007) - angle in degrees $=180 / \pi \cos ^{-1}\left(\operatorname{aspect~ratio}{ }^{-1}\right)$. Using that equation, we

430 calculate that circumferential canals are angled in excess of $45^{\circ}$ and range between $70.5^{\circ}$ to $90^{\circ}$

431 from the longitudinal axis. This discrepancy suggests that circumferential canals are not as

432 adapted to torsion as originally thought. Instead, canals once classified as "longitudinal" may be

433 better torsion-resisting features. For example, we estimate that "longitudinal canals" with the

434 aspect ratio of 1.41 would be aligned with peak principal strains in bone under torsion. The

435 revised biomechanical interpretation may explain why "longitudinal canals" are so abundant not

436 only in homing pigeons but generally in amniotes. Further testing is needed but requires shifts

437 from: (1) assumptions to experiments; (2) two-dimensional to three-dimensional imaging; and

438 (3) categorical to continuous data.

439 The developmental approach used by the current study may inform how the

440 nanostructural organization of collagen fibers also contribute to the torsional rigidity of bone. In

441 adult birds, collagen fibers with oblique-to-transverse orientation (i.e., spiraling $45^{\circ}-90^{\circ}$ to the

442 longitudinal axis) are especially abundant throughout the cortex of bones shaped to resist torsion

443 (de Margerie et al., 2005). Similar collagen fiber orientation evolved independently in adult birds

444 and at least one species of fruit bat (Skedros \& Doutré, 2019) suggesting that it may be a

445 fundamental adaptation of vertebrate flapping flight. If so, we expect collagen fiber obliquity and

446 torsional rigidity of wing bones to increase with locomotor maturity. Preliminary evidence

447 suggests that the predicted trend occurs at least in the ulna of growing turkey (Skedros et al.,

448 2003; Skedros \& Hunt, 2004). Future investigations should apply the developmental approach

449 across a broader phylogenetic sample to test whether loading has similar effects on collagen

450 fibers and vascular canals. 
451

452 Conclusions

453 The altricial limb bones of the homing pigeon show that some structural changes in midshaft

454 cortical bone are predictable responses to delayed locomotor development. During postnatal

455 growth, midshaft cortical geometry scales with positive allometry. Adults appear to have

456 disproportionately stronger limb bones than juveniles consistent with the transition from limited

457 to full mobility. Positive allometric growth is not exclusive to altriciality or precociality. Instead,

458 it probably indicates reduced selection on locomotor performance in juveniles. As their limb

459 bones develop midshaft cortical geometries that are increasingly rigid to locomotor-induced

460 torsion, laminarity decreases. Because locomotor-induced torsion is likely greater in adults than

461 juveniles, our results suggest that low laminarity may actually be a torsion-resisting feature.

462 Bone with low laminarity contains abundant longitudinal canals that may be aligned with peak

463 principal strains in bone loaded under torsion. Future directions include using microtomography

464 and spherical statistics to assess how closely vascular canals approximate peak principal strain

465 direction during growth.

466

467 Acknowledgements

468 We thank Stromberg's Chicks and Gamebirds for access to their salvaged pigeons. Erin Simons

469 provided access to shared lab supplies and equipment. Ravi Bhadriraju, who at the time was a

470 research volunteer and federal work study student, prepared slides of the femur and tibiotarsus.

471 Volumetric renders in Figures 1 and 2 were generated from microCT scanning performed by

472 Baker Hughes Inspection Technologies and Arizona State University 4D Materials Science

473 Center as well as from CT scanning performed by Sasha Willis at Midwestern University’s

474 Companion Animal Clinic. We thank Holly Woodward Ballard, Edina Prondvai, and John 
475 Skedros for their constructive reviews of the manuscript. This is Arizona Research Collection for 476 Integrative Vertebrate Education and Study (ARCIVES) contribution no. XXXX.

477 


\section{References}

479 Altimiras J, Lindgren I, Giraldo-Deck LM, Matthei A, Garitano-Zavala Á. 2017. Aerobic

480 performance in tinamous is limited by their small heart. A novel hypothesis in the

481 evolution of avian flight. Scientific Reports 7:15964. DOI: 10.1038/s41598-017-16297-2.

482

483

484

485

486

487

488

489

490

491

492

493

494

495

496

497

498

499

500

501

Bennett MB. 2008. Post-hatching growth and development of the pectoral and pelvic limbs in the black noddy, Anous minutus. Comparative Biochemistry and Physiology Part A: Molecular \& Integrative Physiology 150:159-168. DOI: 10.1016/j.cbpa.2006.06.011.

Bennett MB, Forwood MR. 2010. Histomorphometric changes in the wing bones of the fruit bat, Pteropus poliocephalus, (Megachiroptera: Pteropidae [sic Pteropodidae]) in relation to increased bone strain and the failure of a good (?) hypothesis. Australian Zoologist $35: 341-348$.

Biewener AA, Bertram JEA. 1994. Structural response of growing bone to exercise and disuse. Journal of Applied Physiology 76:946-955.

Biewener AA, Dial KP. 1995. In vivo strain in the humerus of pigeons (Columba livia). Journal of Morphology 225:61-75. DOI: 10.1002/jmor.1052250106.

Biewener AA, Swartz SM, Bertram JEA. 1986. Bone modeling during growth: dynamic strain equilibrium in the chick tibiotarsus. Calcified Tissue International 39:390-395.

de Boef M, Larsson HCE. 2007. Bone microstructure: quantifying bone vascular orientation. Canadian Journal of Zoology 85:63-70.

Carrano MT, Biewener AA. 1999. Experimental alteration of limb posture in the chicken (Gallus gallus) and its bearing on the use of birds as analogs for dinosaur locomotion. Journal of Morphology 240:237-249.

Carrier DR. 1983. Postnatal ontogeny of the musculo-skeletal system in the Black-tailed jack rabbit (Lepus californicus). Journal of Zoology 201:27-55. 
502 Carrier D, Leon LR. 1990. Skeletal growth and function in the California gull (Larus 503 californicus). Journal of Zoology 222:375-389.

504 Carter DR, Spengler DM. 1978. Mechanical properties and composition of cortical bone.

$505 \quad$ Clinical Orthopaedics and Related Research 135:192-217.

506 Castanet J, Croci S, Aujard F, Perret M, Cubo J, de Margerie E. 2004. Lines of arrested growth 507 in bone and age estimation in a small primate: Microcebus murinus. Journal of Zoology $508 \quad 263: 31-39$.

509 Coles BH. 2007. Appendix 9: Incubation and fledging periods of selected birds. In: Essentials of $510 \quad$ Avian Medicine and Surgery. Blackwell Publishing Ltd, 355-356.

511 Cooper DML, Erickson B, Peele AG, Hannah K, Thomas CDL, Clement JG. 2011. Visualization 512 of 3D osteon morphology by synchrotron radiation micro-CT. Journal of Anatomy 513 219:481-489. DOI: 10.1111/j.1469-7580.2011.01398.x.

514 Cooper D, Turinsky A, Sensen C, Hallgrimsson B. 2003. Quantitative 3D analysis of the canal 515 network in cortical bone by micro computed tomography. The Anatomical Record Part $516 \quad$ B: The New Anatomist 274:169-179.

517 Craig RR Jr. 2000. Mechanics of Materials. New York: John Wiley \& Sons, Inc.

518 Currey JD. 1960. Differences in the blood-supply of bone of different histological types. 519 Quarterly Journal of Microscopical Sciences 101:351-370.

520 Daegling DJ. 2002. Estimation of torsional rigidity in primate long bones. Journal of Human $521 \quad$ Evolution 43:229-239. DOI: 10.1006/jhev.2002.0574.

522 Dial TR, Carrier DR. 2012. Precocial hindlimbs and altricial forelimbs: partitioning ontogenetic 523 strategies in mallards (Anas platyrhynchos). The Journal of Experimental Biology 215:3703-3710. DOI: 10.1242/jeb.057380. 
525 Dial KP, Jackson BE. 2011. When hatchlings outperform adults: locomotor development in 526 Australian brush turkeys (Alectura lathami, Galliformes). Proceedings of the Royal

527

528

529

530

531

532

533

534

535

536

537

538

539

540

541

542

543

544

545

546 Society B 278:1610-1616. DOI: 10.1098/rspb.2010.1984.

Doube M, Kłosowski MM, Arganda-Carreras I, Cordelières FP, Dougherty RP, Jackson JS, Schmid B, Hutchinson JR, Shefelbine SJ. 2010. BoneJ: Free and extensible bone image analysis in ImageJ. Bone 47:1076-1079. DOI: 10.1016/j.bone.2010.08.023.

Douma JC, Weedon JT. 2019. Analysing continuous proportions in ecology and evolution: A practical introduction to beta and Dirichlet regression. Methods in Ecology and Evolution 10:1412-1430. DOI: 10.1111/2041-210X.13234.

Eurell JAC, Sterchi DL. 1994. Microwaveable toluidine blue stain for surface staining of undecalcified bone sections. Journal of Histotechnology 17:357-359. DOI: 10.1179/his.1994.17.4.357.

Fekedulegn BD, Colbert JJ, Hicks Jr. RR, Schuckers ME. 2002. Coping with multicollinearity: an example on application of principal components regression in dendroecology. Newtown Square, Pennsylvania: U.S. Department of Agriculture, Forest Service, Northeastern Research Station.

Ferrari S, Cribari-Neto F. 2004. Beta regression for modelling rates and proportions. Journal of Applied Statistics 31:799-815. DOI: 10.1080/0266476042000214501.

Francillon-Vieillot H, de Buffrénil V, Castanet J, Geraudie J, Meunier FJ, Sire JY, Zylberberg L, de Ricqlès AJ. 1990. Microstructure and mineralization of vertebrate skeletal tissues. In: Carter JG ed. Skeletal Biomineralization: Patterns, Processes and Evolutionary Trends. New York: Van Nostrand Reinhold, 471-548. 
547 Habib MB, Ruff CB. 2008. The effects of locomotion on the structural characteristics of avian $548 \quad$ limb bones. Zoological Journal of the Linnean Society 153:601-624.

549 Hayward JL, Henson SM, Banks JC, Lyn SL. 2009. Mathematical modeling of appendicular 550 bone growth in glaucous-winged gulls. Journal of Morphology 270:70-82. DOI:

551 10.1002/jmor.10669.

552 Hedrick BP, Dickson BV, Dumont ER, Pierce SE. 2020. The evolutionary diversity of locomotor 553 innovation in rodents is not linked to proximal limb morphology. Scientific Reports

554 10:717. DOI: 10.1038/s41598-019-57144-w.

555

556

557

558

559

560

561

562

563

564

565

566

567

568

Hubert M, Rousseeuw P, Verdonck T. 2009. Robust PCA for skewed data and its outlier map. Computational Statistics \& Data Analysis 53:2264-2274. DOI: 10.1016/j.csda.2008.05.027.

Huttenlocker AK, Woodward HN, Hall BK. 2013. The biology of bone. In: Padian K, Lamm ET eds. Bone Histology of Fossil Tetrapods: Advancing Methods, Analysis, and Interpretation. Berkeley, California: University of California Press, 13-34.

Janiga M, Kocian L. 1985. The knowledge of postnatal development of the feral pigeon (Columba livia f. domestica) in Bratislava. Biológia (Bratislava) 40:189-197.

Johnston RF, Janiga M. 1995. Feral Pigeons. New York: Oxford University Press.

Kilmer JT, Rodríguez RL. 2017. Ordinary least squares regression is indicated for studies of allometry. Journal of Evolutionary Biology 30:4-12. DOI: 10.1111/jeb.12986.

Kuehn AL, Lee AH, Main RP, Simons ELR. 2019. The effects of growth rate and biomechanical loading on bone laminarity within the emu skeleton. PeerJ 7:e7616. DOI: $10.7717 /$ peerj.7616. 
569 Lanyon LE, Rubin CT. 1984. Static vs. dynamic loads as an influence on bone remodeling.

$570 \quad$ Journal of Biomechanics 17:897-905.

571 Lee AH, O’Connor PM. 2013. Bone histology confirms determinate growth and small body size 572 in the noasaurid theropod Masiakasaurus knopfleri. Journal of Vertebrate Paleontology 573 33:865-876. DOI: 10.1080/02724634.2013.743898.

574 Lee AH, Simons ELR. 2015. Wing bone laminarity is not an adaptation for torsional resistance 575 in bats. PeerJ 3:e823. DOI: 10.7717/peerj.823.

576

577

578

579

580

581

582

583

584

585

586

587

588

589

590

591

Legendre LJ, Bourdon E, Scofield RP, Tennyson AJD, Lamrous H, de Ricqlès A, Cubo J. 2014. Bone histology, phylogeny, and palaeognathous birds (Aves: Palaeognathae). Biological Journal of the Linnean Society 112:688-700. DOI: 10.1111/bij.12312.

Levi WM. 1962. The Pigeon. Sumter, SC: Levi Publishing.

Liang X, Yu J, Wang H, Zhang Z. 2018. Post-hatching growth of the pectoralis muscle in pigeon and its functional implications. The Anatomical Record 301:1564-1569. DOI: 10.1002/ar.23850.

Main RP. 2007. Ontogenetic relationships between in vivo strain environment, bone histomorphometry and growth in the goat radius. Journal of Anatomy 210:272-293.

Main RP, Biewener AA. 2004. Ontogenetic patterns of limb loading, in vivo bone strains and growth in the goat radius. Journal of Experimental Biology 207:2577-2588.

Main RP, Biewener AA. 2006. In vivo bone strain and ontogenetic growth patterns in relation to life-history strategies and performance in two vertebrate taxa: goats and emu. Physiological and Biochemical Zoology 79:57-72.

Main RP, Biewener AA. 2007. Skeletal strain patterns and growth in the emu hindlimb during ontogeny. Journal of Experimental Biology 210:2676. 
592 de Margerie E. 2002. Laminar bone as an adaptation to torsional loads in flapping flight. Journal 593 of Anatomy 201:521-526. DOI: 10.1046/j.1469-7580.2002.00118.x.

594 de Margerie E, Cubo J, Castanet J. 2002. Bone typology and growth rates: testing and 595 quantifying “Amprino's rule" in the mallard (Anas platyrhynchos). Comptes Rendus 596 Biologies 325:221-230. DOI: 10.1016/S1631-0691(02)01429-4.

597 de Margerie E, Robin J-P, Verrier D, Cubo J, Groscolas R, Castanet J. 2004. Assessing a 598 relationship between bone microstructure and growth rate: a fluorescent labelling study in 599

600 the king penguin chick (Aptenodytes patagonicus). Journal of Experimental Biology 207:869-879.

601 602 603 604 605 606 607 608 609 610 611 612 613 614

de Margerie E, Sanchez S, Cubo J, Castanet J. 2005. Torsional resistance as a principal component of the structural design of long bones: comparative multivariate evidence in birds. Anatomical Record 282A:49-66. DOI: 10.1002/ar.a.20141.

Pennycuick CT. 1967. The strength of the pigeon's wing bones in relation to their function. Journal of Experimental Biology 46:219-233.

Ponton F, Elzanowski A, Castanet J, Chinsamy A, de Margerie E, de Ricqlès AJ, Cubo J. 2004. Variation of the outer circumferential layer in the limb bones of birds. Acta Ornithologica 39:137-140.

Pratt IV, Cooper DML. 2017. A method for measuring the three-dimensional orientation of cortical canals with implications for comparative analysis of bone microstructure in vertebrates. Micron 92:32-38. DOI: 10.1016/j.micron.2016.10.006.

Pratt IV, Johnston JD, Walker E, Cooper DML. 2018. Interpreting the three-dimensional orientation of vascular canals and cross-sectional geometry of cortical bone in birds and bats. Journal of Anatomy 232:931-942. DOI: 10.1111/joa.12803. 
615 R Core Team. 2019. R: A language and environment for statistical computing. Vienna, Austria:

$616 \quad$ R Foundation for Statistical Computing.

617 Ren Y, Wang H, Zhang Z. 2016. Postnatal variation of limb bones in the Japanese quail, 618 Coturnix coturnix japonica. International Journal of Morphology 34:708-712.

619 Reynkens T. 2018. rospca: Robust Sparse PCA using the ROSPCA Algorithm.

620 de Ricqlès AJ, Meunier FJ, Castanet J, Francillon-Vieillot H. 1991. Comparative microstructure 621 of bone. In: Hall BK ed. Bone, Volume 3: Bone Matrix and Bone Specific Products.

622 Boston: CRC Press, 1-78.

623 Rigby RA, Stasinopoulos DM. 2005. Generalized additive models for location, scale and shape. $624 \quad$ Applied Statistics 54:507-554.

625 Ruff CB. 2002. Long bone articular and diaphyseal structure in old world monkeys and apes. I:

626 Locomotor effects. American Journal of Physical Anthropology 119:305-342. DOI:

627 10.1002/ajpa.10117.

628

Ruff CB, Burgess ML, Bromage TG, Mudakikwa A, McFarlin SC. 2013. Ontogenetic changes in 629 limb bone structural proportions in mountain gorillas (Gorilla beringei beringei). Journal

630 of Human Evolution 65:693-703.

631 Sales J. 2007. The emu (Dromaius novaehollandiae): a review of its biology and commercial 632 products. Avian and Poultry Biology Reviews 18:1-20. DOI:

633 $10.3184 / 135704807 X 222531$.

634 Schoenau E, Neu CM, Rauch F, Manz F. 2001. The development of bone strength at the 635 proximal radius during childhood and adolescence. The Journal of Clinical 636 Endocrinology \& Metabolism 86:613-618. DOI: 10.1210/jcem.86.2.7186. 
637 Simons ELR, O’Connor PM. 2012. Bone laminarity in the avian forelimb skeleton and its

638 relationship to flight mode: testing functional interpretations. The Anatomical Record

639 295:386-396. DOI: 10.1002/ar.22402.

640 Skedros JG, Doutré MS. 2019. Collagen fiber orientation pattern, osteon morphology and

641 distribution, and presence of laminar histology do not distinguish torsion from bending in

642 bat and pigeon wing bones. Journal of Anatomy 234:748-763. DOI: 10.1111/joa.12981.

643 Skedros JG, Hunt KJ. 2004. Does the degree of laminarity correlate with site specific differences

644 in collagen fibre orientation in primary bone? An evaluation in the turkey ulna diaphysis.

645 Journal of Anatomy 205:121-134. DOI: 10.1111/j.0021-8782.2004.00318.x.

646 Skedros JG, Hunt KJ, Hughes PE, Winet H. 2003. Ontogenetic and regional morphological

647 variations in the turkey ulna diaphysis: implications for functional adaptation of cortical

648 bone. Anatomical Record 273A:609-629.

649 Smithson M, Verkuilen J. 2006. A better lemon squeezer? Maximum-likelihood regression with

650 beta-distributed dependent variables. Psychological Methods 11:54-71. DOI:

$651 \quad 10.1037 / 1082-989 X .11 .1 .54$

652 Starck JM, Ricklefs RE. 1998. Patterns of development: the altricial-precocial spectrum. In:

653 Avian growth and development. Evolution within the altricial-precocial spectrum. New

654 York: Oxford University Press, 3-30.

655 Swartz SM, Bennett MB, Carrier DR. 1992. Wing bone stresses in free flying bats and the 656 evolution of skeletal design for flight. Nature 359:726-729. DOI: 10.1038/359726a0.

657 Tobalske B, Dial K. 1996. Flight kinematics of black-billed magpies and pigeons over a wide $658 \quad$ range of speeds. Journal of Experimental Biology 199:263-280.

659 Vriends MM, Erskine TE. 2005. Pigeons. New York: Barron’s Educational Series, Inc. 
660 Young JW, Fernández D, Fleagle JG. 2010. Ontogeny of long bone geometry in capuchin 661 monkeys (Cebus albifrons and Cebus paella): implications for locomotor development 662 and life history. Biology Letters 6:197-200. DOI: 10.1098/rsbl.2009.0773.

663 Zioupos P, Cook RB, Hutchinson JR. 2008. Some basic relationships between density values in 664 cancellous and cortical bone. Journal of Biomechanics 41:1961-1968. DOI:

$665 \quad$ 10.1016/j.jbiomech.2008.03.025.

666 


\section{Figure 1}

Cross-sectional profile of a primary vascular canal is a useful approximation for canal orientation.

(A) The histological view of the dorsal octant of ulna from a post-fledge homing pigeon (MWU 257) was imaged from an undecalcified $100-\mu \mathrm{m}$ transverse section stained with toluidine blue. The stain highlights edges of primary vascular canals at the plane of section, allowing accurate characterization of the cross-sectional profile of each canal. Canals with nearly circular profiles (aspect ratio < 3) have a longitudinal orientation (blue arrow), whereas those with greatly elongated profiles have a transverse orientation (red arrow). Whether the transverse orientation is specifically circumferential, radial, or oblique depends on how much the long axis of the canal profile is angled relative to the periosteal surface. This method of estimating three-dimensional orientation from two-dimensional canal profile assumes that canal shape is generally cylindrical. (B) MicroCT view ( 2- $\mu \mathrm{m}$ voxel resolution) of the same specimen about $1 \mathrm{~mm}$ from the plane of histological section reveals circular (blue arrow) and elongated (red arrow) cross-sectional profiles of canals. (C) Three-dimensional rendering of canals (yellow) confirms that circular (blue arrow) and elongated (red arrow) profiles accurately reflect orientation-longitudinal and circumferential, respectively. MicroCT imaging was performed using a Zeiss Versa 520 at Arizona State University 4D Materials Science Center and was rendered with Avizo Lite (9.0.1). 


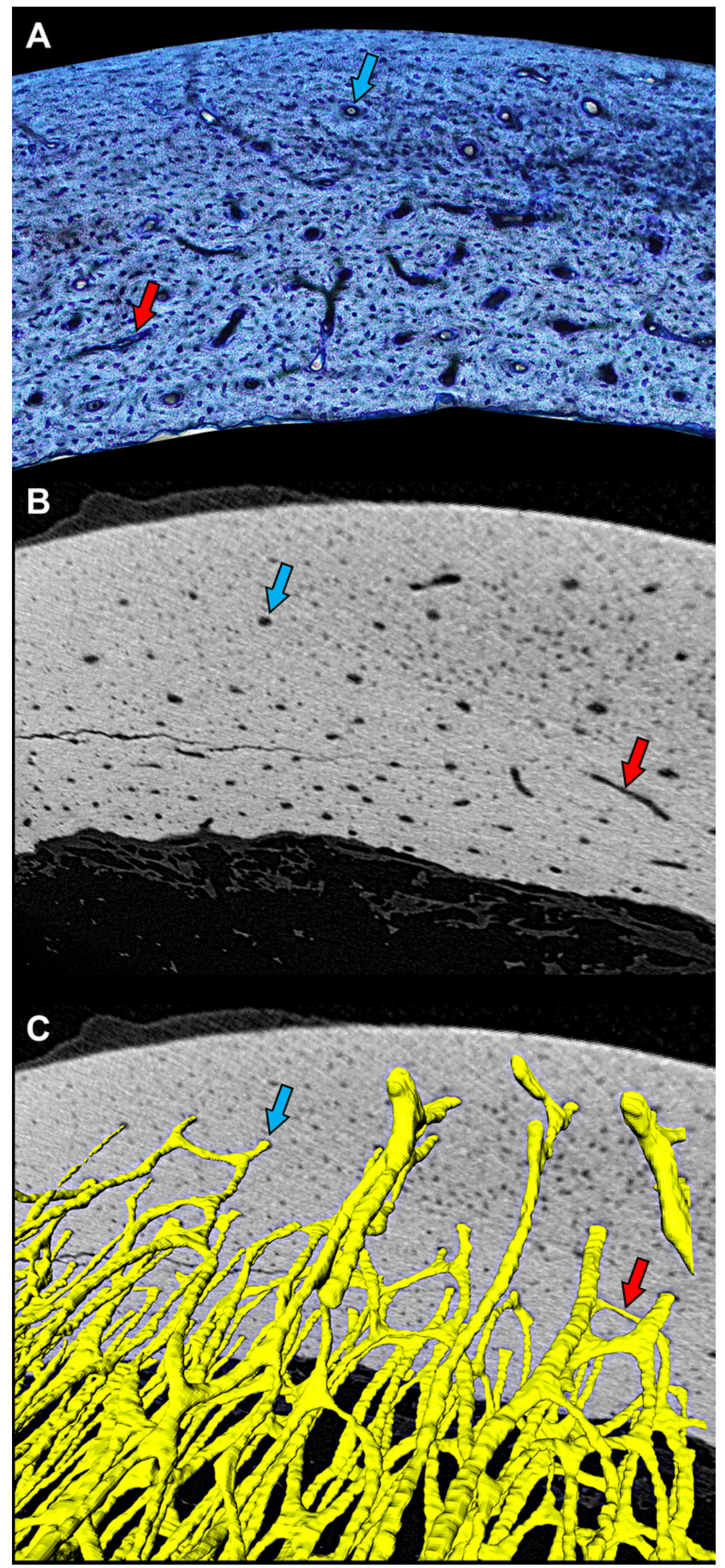

Peer] reviewing PDF | (2020:03:46738:2:0:NEW 6 Aug 2020) 


\section{Figure 2}

Preparation of bone profile to evaluate laminarity index.

(A) The five listed bone elements were harvested from a growth series of 17 pigeons.

Undecalcified and stained histological sections were taken from midshaft. For graphical illustration, MWU 269 was imaged using a Siemens SOMATOM Perspective CT scanner, and the left-sided elements were visualized with Avizo Lite. (B) Each histological section was divided into four octants representing cardinal anatomical positions based on a posture in which the pigeon is standing with the forelimbs extended laterally (i.e., cranial, caudal, dorsal, and ventral for the wing elements; cranial, caudal medial, and lateral for the hindlimb elements). Octant curvature (C) was straightened (D) using ImageJ. (E) Canals were fit with ellipses and classified based on orientation relative to the horizontal periosteal surface. 


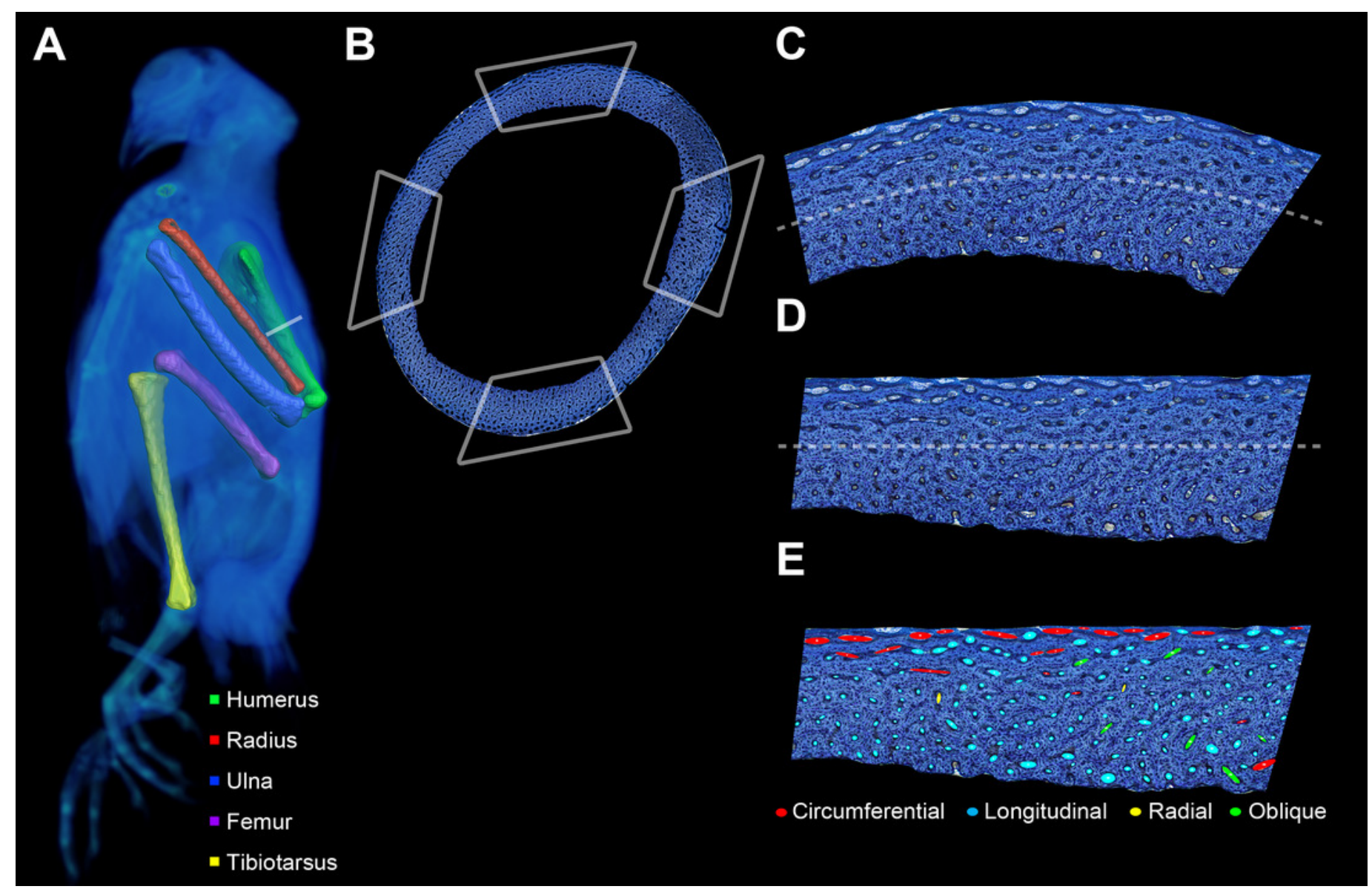




\section{Figure 3}

Histology of representative bone elements from a growth series of homing pigeons arranged by mass.

In ascending order (bottom to top): MWU 271 (209 g, 3-4 weeks); MWU 258 (242 g, 2-3 weeks); MWU 273 (314 g, 4-5 weeks); MWU 256 (455 g, 5-6 weeks); MWU 254 (482 g, 8-10 weeks). Cortical bone porosity decreases with mass and age. Circumferential vascular canals are most abundant in the humerus, ulna, and femur of pre-fledge juveniles (< 5 weeks).

Scale bar equals $600 \mu \mathrm{m}$ (A,B,D-F \& K), $480 \mu \mathrm{m}$ (G,L \& M), $400 \mu \mathrm{m}(\mathbf{I}, \mathbf{J}, \mathbf{N}-\mathbf{P}), 343 \mu \mathrm{m}$ (Q,T$\mathbf{V})$, and $300 \mu \mathrm{m}(\mathbf{C}, \mathbf{H}, \mathbf{R}, \mathbf{S}, \mathbf{W}-\mathbf{Y})$. Digital slides are freely accessible at http://paleohistology.appspot.com/Page/Columba livia.html . 


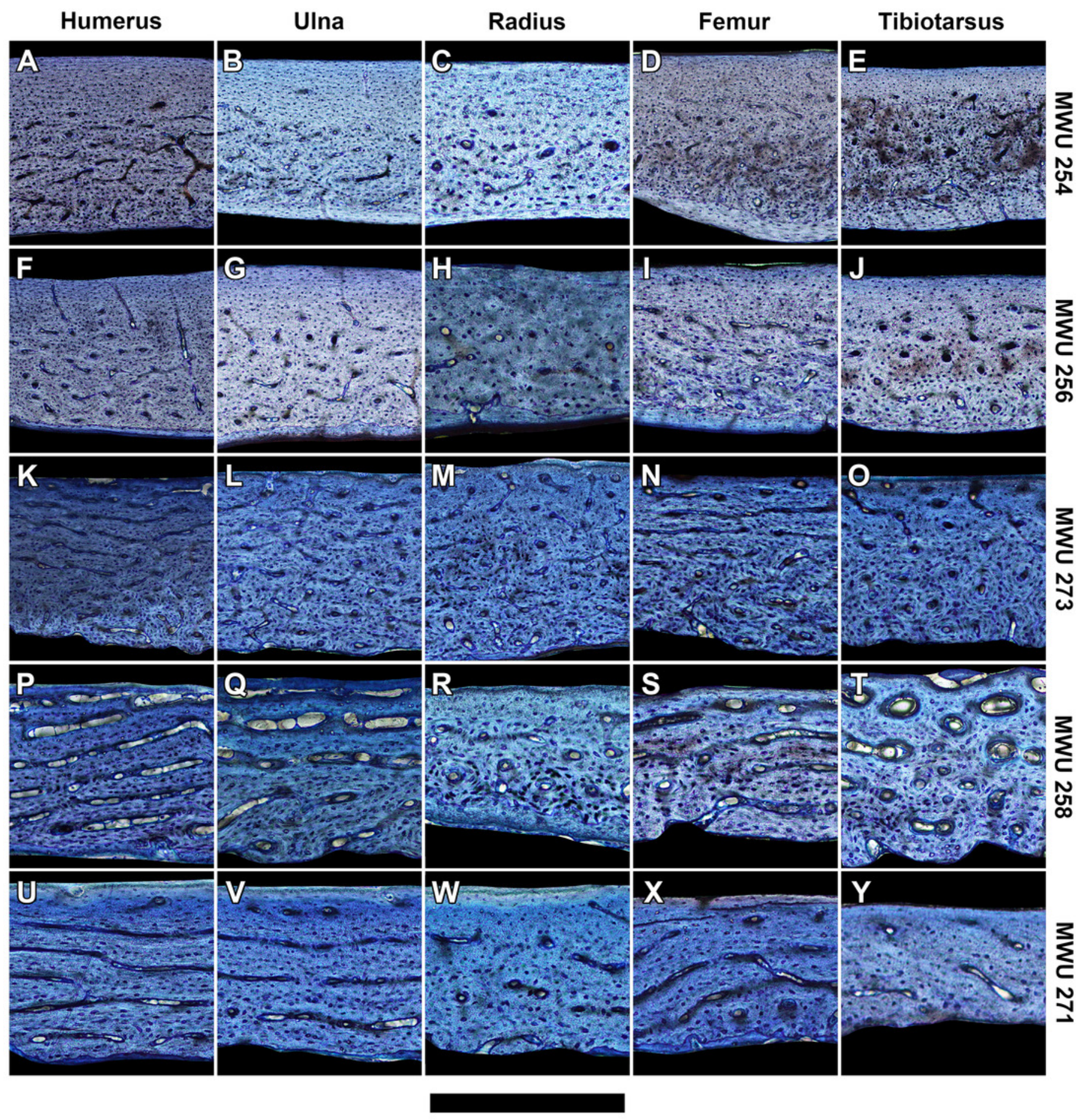




\section{Figure 4}

Positive allometric scaling of polar section modulus $\left(Z_{p}\right)$ in an ontogenetic series of (A) humerus, (B) ulna, $(C)$ radius, $(D)$ femur, and (E) tibiotarsus from the homing pigeon.

Colored lines represent the relationship between $\log _{10}$-transformed body mass and $Z_{p}$ in the form: $\log _{10}(Z p)=\log _{10}(b)+a \log _{10}($ body mass $)$. Shaded regions are $95 \%$ confidence bands. Solid black lines indicate the slope of isometry for reference. 

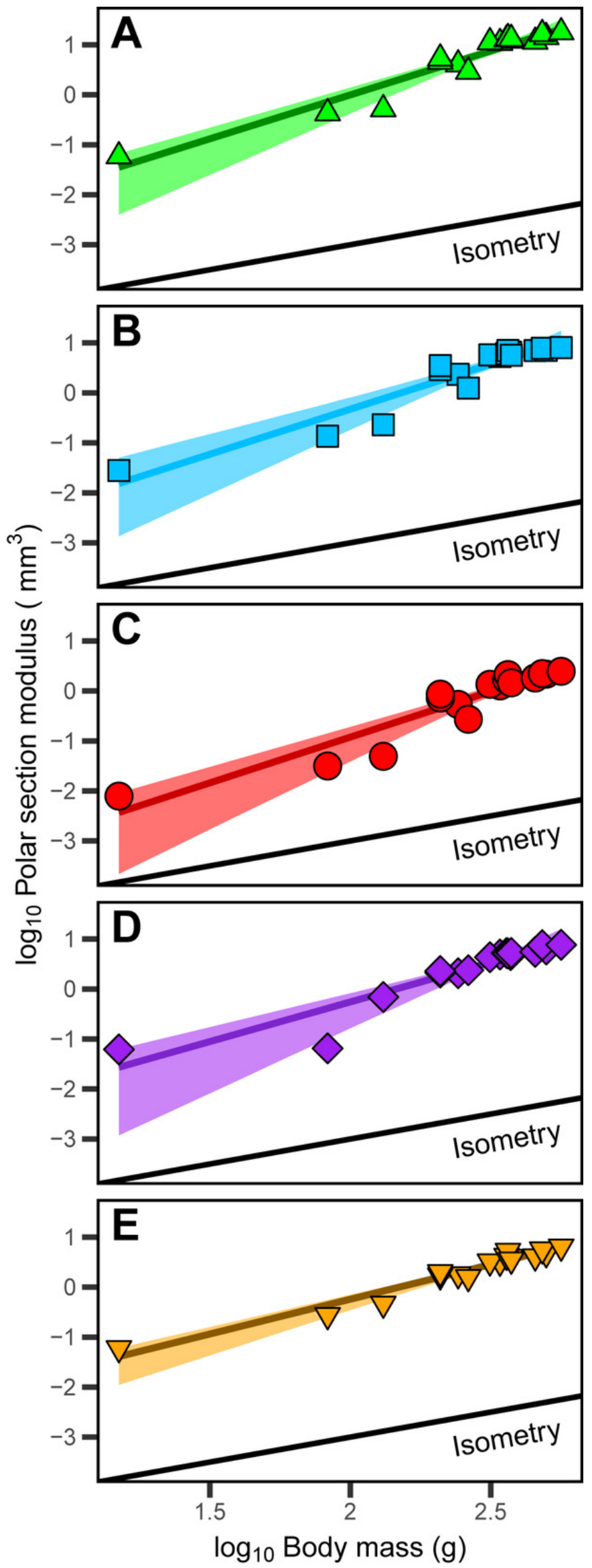

Peer] reviewing PDF | (2020:03:46738:2:0:NEW 6 Aug 2020) 


\section{Figure 5}

Relationship between laminarity (LI) and the "ontogenetic axis" of variation.

Fitted beta regression models were back-transformed using the inverse logit function to ease interpretation of the ontogenetic trends in laminarity on the scale of 0 to 1 . The back-

transformed curves follow Eq. 3: $\mathrm{LI}=\exp \left(\beta_{0}+\beta_{1} \mathrm{PC} 1\right) /\left(1+\exp \left(\beta_{0}+\beta_{1} \mathrm{PC} 1\right)\right)$. For each unit of increase along $\mathrm{PCl}$ (the "ontogenetic axis"), laminarity decreases non-linearly given the following values of $\beta_{0}$ and $\beta_{1}$ : (A) -1.283 and -0.249 in the humerus; (B) -1.670 and -0.245 in the ulna; (C) -2.585 and -0.197 in the radius; (D) -1.564 and -0.244 in the femur; and (E) -2.657 and -0.137 in the tibiotarsus.
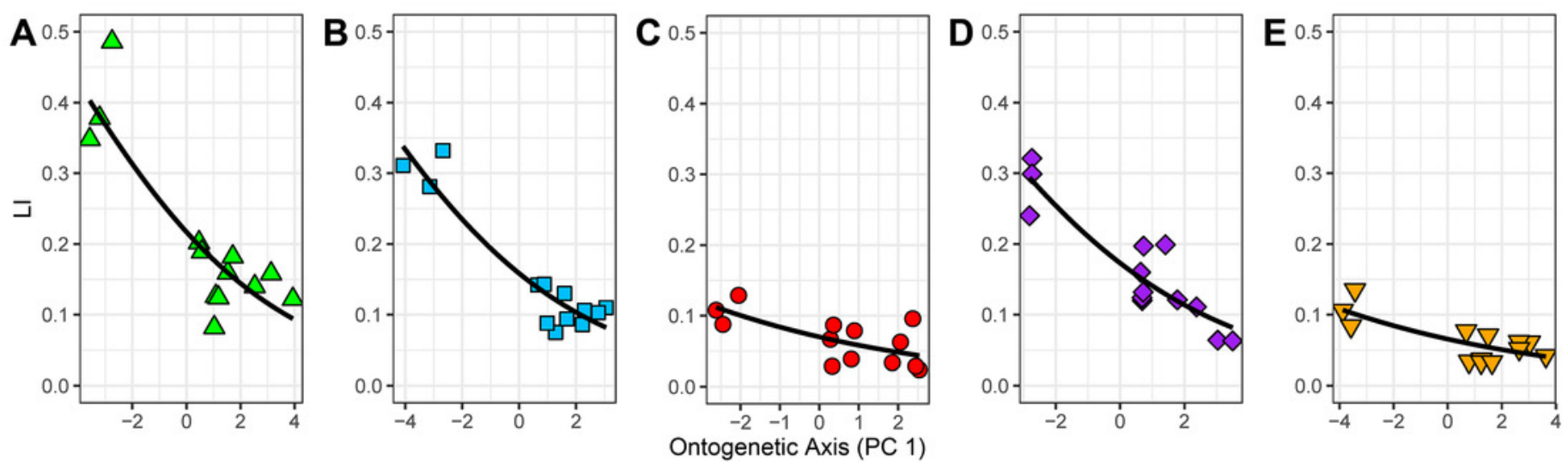


\section{Table $\mathbf{1}$ (on next page)}

Ontogenetic scaling of $\log _{10}$-transformed polar section modulus in the homing pigeon.

Parameters of linear regression fit are presented in $\log _{10}$ scale. Isometry is equivalent to a scaling exponent (a) of 1. 
1 Table 1:

2 Ontogenetic scaling of $\log _{10}$-transformed polar section modulus in the homing pigeon.

3 Parameters of linear regression fit are presented in $\log _{10}$ scale. Isometry is equivalent to a scaling 4 exponent $(a)$ of 1 .

5

\begin{tabular}{|l|c|c|c|c|c|}
\hline Element & $\boldsymbol{R}^{\mathbf{2}}$ & $\boldsymbol{a}$ & $\mathbf{9 5 \%}$ CI & $\boldsymbol{b}$ & $\mathbf{9 5 \%}$ CI \\
\hline Humerus $(n=17)$ & 0.93 & 1.76 & $1.58,2.46$ & -3.52 & $-5.29,-3.04$ \\
\hline Ulna $(n=17)$ & 0.90 & 1.80 & $1.46,2.59$ & -3.93 & $-4.74,-3.12$ \\
\hline Radius $(n=17)$ & 0.88 & 1.83 & $1.59,2.73$ & -4.58 & $-6.86,-3.92$ \\
\hline Femur $(n=17)$ & 0.87 & 1.59 & $1.34,2.61$ & -3.43 & $-5.99,-2.79$ \\
\hline Tibiotarsus $(n=17)$ & 0.95 & 1.39 & $1.28,1.82$ & -3.02 & $-4.09,-2.73$ \\
\hline
\end{tabular}

6 
Table 2 (on next page)

Results from robust principal component analysis (PCA). 


Table 2:
2 Results from robust principal component analysis (PCA).
\begin{tabular}{|l|l|c|c|c|}
\hline Element & \multicolumn{1}{l|}{ PC1 } & PC2 & PC3 \\
\hline Humerus & Eigenvalues & 5.384 & 0.100 & 0.022 \\
& Standard deviation & 2.320 & 0.316 & 0.148 \\
& Proportion of variance & 0.978 & 0.018 & 0.004 \\
& Mass eigenvector & 0.262 & -0.440 & 0.859 \\
& Length eigenvector & 0.522 & 0.813 & 0.258 \\
& $Z_{p}$ eigenvector & 0.812 & -0.381 & -0.443 \\
\hline \multirow{5}{*}{ Ulna } & Eigenvalues & 5.581 & 0.115 & 0.048 \\
& Standard deviation & 2.362 & 0.339 & 0.218 \\
& Proportion of variance & 0.972 & 0.020 & 0.008 \\
& Mass eigenvector & 0.274 & 0.896 & -0.350 \\
& Length eigenvector & 0.797 & -0.415 & -0.438 \\
& $Z_{p}$ eigenvector & 0.538 & 0.159 & 0.828 \\
\hline Radius & Eigenvalues & 3.184 & 0.168 & 0.018 \\
& Standard deviation & 1.784 & 0.409 & 0.133 \\
& Proportion of variance & 0.945 & 0.050 & 0.005 \\
& Mass eigenvector & 0.453 & 0.689 & -0.566 \\
& Length eigenvector & 0.817 & -0.575 & -0.046 \\
& $Z_{p}$ eigenvector & 0.357 & 0.441 & 0.823 \\
\hline Femur & Eigenvalues & 3.963 & 0.047 & 0.038 \\
& Standard deviation & 1.991 & 0.217 & 0.196 \\
& Proportion of variance & 0.979 & 0.012 & 0.009 \\
& Mass eigenvector & 0.348 & 0.845 & 0.407 \\
& Length eigenvector & 0.689 & -0.525 & 0.499 \\
& $Z_{p}$ eigenvector & 0.635 & 0.107 & -0.765 \\
\hline & Eigenvalues & 6.706 & 0.233 & 0.048 \\
& Standard deviation & 2.290 & 0.483 & 0.218 \\
& Proportion of variance & 0.960 & 0.033 & 0.007 \\
& Mass eigenvector & 0.286 & 0.480 & -0.830 \\
& Length eigenvector & 0.867 & -0.499 & 0.010 \\
& $Z_{p}$ eigenvector & 0.409 & 0.722 & 0.558 \\
\hline
\end{tabular}

4 


\section{Table 3 (on next page)}

Relationship between laminarity and scores of $\mathrm{PC} 1$ using beta regression with a logit link.

Standardized coefficients for each of the original variables (mass, $Z_{p}$, and bone length) are also listed. 


\section{Table 3:}

2 Relationship between laminarity and scores of PC1 using beta regression with a logit link.

3

\begin{tabular}{|l|c|c|c|c|c|ccc|}
\hline & & & & & \multicolumn{3}{|c|}{ Standardized Coefficients } \\
\cline { 6 - 9 } Element & Pseudo & $\boldsymbol{\beta}_{\mathbf{0}}$ & $\mathbf{p}-$-value & $\boldsymbol{\beta}_{\mathbf{1}}$ & p-value & Mass & Length & $\boldsymbol{Z}_{\boldsymbol{p}}$ \\
& $\mathbf{R}^{\mathbf{2}}$ & & & & & & & \\
\hline Humerus & 0.726 & -1.283 & $9.47 \mathrm{e}-8$ & -0.249 & $1.22 \mathrm{e}-4$ & -0.065 & -0.130 & -0.202 \\
Radius & 0.440 & -2.585 & $5.7 \mathrm{e}-10$ & -0.197 & 0.007 & -0.089 & -0.161 & -0.070 \\
Ulna & 0.852 & -1.670 & $2.0 \mathrm{e}-10$ & -0.245 & $2.29 \mathrm{e}-6$ & -0.067 & -0.195 & -0.131 \\
Femur & 0.819 & -1.564 & $4.9 \mathrm{e}-10$ & -0.244 & $1.31 \mathrm{e}-5$ & -0.085 & -0.168 & -0.155 \\
Tibiotarsus & 0.521 & -2.657 & $5.0 \mathrm{e}-11$ & -0.137 & 0.002 & -0.039 & -0.119 & -0.056 \\
\hline
\end{tabular}

4 